\title{
Leptin, Diabetes, and the Obesity-A Corelation
}

\author{
Dr. Anil Batta
}

Professor \& Head, Department of Medical Biochemistry Govt. Medical College, Amritsar, Punjab, India

\author{
DOI: $\underline{10.36348 / \mathrm{sijb} .2020 . \mathrm{v} 03 \mathrm{i} 02.005}$
}

| Received: 13.02.2020 | Accepted: 21.02.2020 | Published: 23.02 .2020

*Corresponding author: Dr. Anil Batta

\section{Abstract}

Obesity is a chronic disease and a major global health challenge. Apart from bariatric surgery, which is costly and not without risk, there are currently no successful long-term treatment options for obesity. The history of pharmacological agents for obesity has been turbulent with many examples of drugs being removed from the market due to significant side effects. Orlistat and sibutramine (the latest drugs on the market) provide only modest weight loss and are both associated with high attrition rates due to intolerable side effects. Furthermore, sibutramine was recently withdrawn from the market. There is a need for the development of safe and efficacious drug treatments for obesity. Methods: From our sample collection of more than 65 subjects for metabolic disorders research. These subjects were grouped on the basis of diabetes, obesity and gender status. Equal number of subjects was taken for each comparison group to minimize the effects of confounding factors. For diabetes, these sixty five subjects were divided into two groups ( $\mathrm{n}=40$ non-diabetic and $n=30$ type 2 diabetics). While considering obesity, these eighty subject were divided on the basis of their Body Mass Index ( $n=35$ non-obese and $n=40$ obese). For gender based analysis, the groups based on diabetes and obesity were made $(n=20$ in each sub-group) and further analyzed while keeping $n=10$ males and $n=10$ females in each sub-group. Serum leptin and biochemical analytes were measured for all subjects and data were analyzed by SPSS 18.0 software. Results: Leptin levels were found significantly low in nonobese as compared to obese subjects (Mean \pm SD: $5.2 \pm 2.0$ vs. $7.1 \pm 2.1 \mathrm{ng} / \mathrm{ml}, \mathrm{p}<0.001)$. No significant difference was found for non-diabetics and diabetics $(6.4 \pm 2.4$ vs. $5.9 \pm 2.2$ $\mathrm{ng} / \mathrm{ml}, \mathrm{p}=0.35)$. Gender dimorphism showed significant differences for leptin $(\mathrm{p}<0.0001)$. Spearman's analysis showed strong correlation between leptin and obesity $(r=0.43, p=0.0001)$, gender $(r=0.24, p=0.02)$ irrespective of obesity or diabetes.

Keywords: Amylin, leptin, metreleptin, obesity, pramlitide.

Copyright @ 2020: This is an open-access article distributed under the terms of the Creative Commons Attribution license which permits unrestricted use, distribution, and reproduction in any medium for non-commercial use (NonCommercial, or CC-BY-NC) provided the original author and source are credited.

\section{INTRODUCTION}

\section{Leptin regulation of glucose homeostasis}

Leptin is a polypeptide hormone produced and secreted by white adipose tissue (WAT) [6] that circulates in proportion to body fat mass [7], enters the CNS in proportion to its plasma level [8] and interacts with its receptor expressed in key brain areas that regulate food intake, energy expenditure, and autonomic function [9]. A large body of evidence suggests that leptin plays a vital role in the regulation of energy homeostasis as conditions characterized by leptin deficiency promote hyperphagia and weight gain [1], whereas administration of leptin leads to reduced food intake, increased energy expenditure, and weight loss $[2,6]$. However, recent evidence implicates leptin not only in the regulation of energy balance but glucose homeostasis as well [4]. Recent studies suggest that the effect of intrahypothalamic infusion of insulin [2] or free fatty acids [3] to improve hepatic insulin sensitivity requires intact vagal input to the liver. Consistent with the hypothesis that a similar mechanism mediates the effect of leptin in the brain to suppress hepatic glucose production, the effect of restored hypothalamic leptin signaling to improve hepatic insulin sensitivity in obese Koletsky rats was blocked by selective hepatic vagotomy [6]. These data support a model whereby leptin activates a neural signal between the brain and the liver, which regulates hepatic insulin action. Leptin levels drop in fasting or starvation state while increase following refeeding. Leptin levels are directly correlated with fat mass and are elevated in obese patients, who are leptin resistant [6]. Due to defect in either secretion of leptin or abnormal leptin there is leptin deficiency which is a hallmark of certain rare cases of obesity. Leptin deficiency or resistance can result in profound obesity and diabetes. Leptin-deficient mice and humans are severely obese and have several metabolic and endocrine alterations, such as hyperglycemia, insulin resistance, hypertriglyceridemia, 
and central hypothyroidism [7]. If the defect is on leptin receptors in the brain or in the downstream signaling pathways, which are the target sites for leptin action; it would not exert its effects and will be unable to regulate food intake and energy homeostasis. In this situation, brain is irresponsive for the intake of food and energy, thus elicits a response to the body for more food intake, which can cause weight gain and obesity. Since obesity is a major risk factor for type 2 diabetes mellitus (T2D), the increase in adipose tissue may lead to diabetes. Obesity or increase in fat mass also alters the level of adipokines like leptin, adiponectin etc. which are released by the adipose tissue, thus it is suggested that such altered level of adipokines are involved in the pathogenesis of obesity associated diabetes. Leptin level increases in obesity and its correlation with body mass index (BMI) and glucose levels has already been reported in different populations [8].

Table-1: Demographic data of all the groups included in the study

\begin{tabular}{|l|l|l|l|l|l|l|l|}
\hline & Group 1 (N=25) & Group 2 (N=25) & $\boldsymbol{t}$-Test & $\boldsymbol{p}$ & $\begin{array}{l}\text { Group 3 } \\
(\mathbf{N}=15)\end{array}$ & Tests & $\boldsymbol{p}$ \\
\hline Age & & & & & & & \\
\hline Mean \pm SD & $44 \pm 6.9$ & $43.3 \pm 6.7$ & 1.09 & $>0.5$ & $42 \pm 2.7$ & 1.12 & $>0.5$ \\
\hline Range & $24-54$ & $33-45$ & & & 24055 & & \\
\hline Sex & & & & & & & \\
\hline Male & $15(54.8)$ & $10(34.9)$ & 1.08 & $>0.5$ & $10(54.8)$ & 0.32 & $>0.5$ \\
\hline Female & $10(34.6)$ & $15(65.8)$ & & & $5(48.9)$ & & \\
\hline BMI & & & & & & & \\
\hline Mean \pm SD & $34.9 \pm 5.4$ & $21.98 \pm 3.1$ & $12 . .9$ & $<0.001$ & $23.87 \pm 4.76$ & 130 & $<0.001$ \\
\hline Range & $26-43$ & $18-28$ & & & $18-35$ & & \\
\hline
\end{tabular}

\section{MATERIALS AND METHODS}

This was a case-control study carried out at Govt. Medical College, Amritsar. Five milliliters of venous blood was drawn from diabetics and control subjects in the morning after an overnight fast of at least $10 \mathrm{~h}$. A drop of the blood was used instantly to measure the FBS levels with the help of a glucometer and glucose test strips. Serum collected from rest of the blood was stored at $-80^{\circ} \mathrm{C}$. Leptin levels were determined using sandwich enzyme-linked immunosorbent assay (ELISA) kit (EIA-2395 Germany). The human leptin concentrations of the controls and cases were determined from the standard curve by matching their mean absorbance readings with the corresponding human leptin concentration. Informed written consent was taken from each one of the 65 subjects enrolled in the study. All the subjects underwent a detailed history, complete physical examination, and relevant laboratory investigations.
Body mass index (BMI) was calculated as weight (kg) divided by height $\left(\mathrm{m}^{2}\right)$. Diabetes was considered to be present when the FBS was $>126 \mathrm{mg} / \mathrm{dl}$ or the patient was on antidiabetic medicine. A person was considered nondiabetic when the FBS was $<101 \mathrm{mg} / \mathrm{dl}$. Exclusion criteria included the subjects from whom the written consent could not be taken, the patients with acute inflammatory disease in the last 15 days (as acute inflammation may lead to hyperleptinemia), or FBS between 101 and $126 \mathrm{mg} / \mathrm{dl}$. Statistical Package for Social Sciences (SPSS) version 15 software was used for statistical analysis. Descriptive statistics (such as mean and standard error) were compared between cases and controls' data. Unpaired $t$-test was used to find out whether there was any statistical significance in between the two groups. Statistical significance was set at the $95 \%$ confidence level $(P<0.05)$. Pearson correlation was used to find out the relation between serum leptin and FBS in diabetics.

Table-2: Demographic data of all the groups included in the study

\begin{tabular}{|c|c|c|c|c|c|c|c|}
\hline Mean \pm SD & $\mathbf{4 4} \pm .98$ & $\mathbf{4 2 . 9} \pm \mathbf{6 . 9}$ & $\mathbf{1 . 0 0 3}$ & $\mathbf{> 0 . 5}$ & $\mathbf{4 2 . 6} \pm \mathbf{6 . 9}$ & $\mathbf{1 . 1 4}$ & $\mathbf{> 0 . 5}$ \\
\hline Range & $21-65$ & $32-43$ & & & $23-54$ & & \\
\hline Sex & & & & & & & \\
\hline Female & $12(31.2)$ & $20(66.7)$ & & & $9(43.0)$ & & \\
\hline BMI & & & & & & & \\
\hline Mean \pm SD & $32.98 \pm 4.7$ & $22 \pm 3.8$ & 12.8 & $<0.001$ & $21.6 \pm 3.87$ & 1.30 & $<0.001$ \\
\hline Range & $23-54$ & $21-34$ & & & $17-31$ & & \\
\hline & & & & & & & \\
\hline
\end{tabular}

Table-3: Fasting blood sugar and serum leptin levels in diabetic and nondiabetic subjects

\begin{tabular}{|c|c|c|c|c|c|}
\hline & NO. OF SUBJECTS & MEAN & SD & SEM & P VALUE \\
\hline FBS (mg \%) & & & & & \\
\hline CONTROL & 60 & 100.3 & 19.121 & 2.987 & 0.001 \\
\hline CASES & 65 & 203.7 & 45.987 & 6.987 & \\
\hline LEPTIN (NG/ML) & & & & & \\
\hline CONTROL & 60 & 15.870 & 16.987 & 2.012 & 0.001 \\
\hline CASE & 65 & 50.87 & 49.98 & 7.654 & \\
\hline
\end{tabular}


Table-6: The lipid profiles including serum cholesterol (mg/dl) and serum triglycerides (mg/dl) of the studied groups

\begin{tabular}{|l|l|l|l|l|l|l|l|}
\hline & Group 1 & Group 2 & $\boldsymbol{t}$-Test & $\boldsymbol{p}$ & Group 3 & $\boldsymbol{F}$-Test (ANOVA) & $\boldsymbol{p}$ \\
\hline TC & & & & & & & \\
\hline Mean \pm SD & $260.9 \pm 56.9$ & $243 \pm 34.8$ & 2.07 & $<0.05$ & $189 \pm 23.8$ & 13.98 & $<0.001$ \\
\hline Range & $180-345$ & $189-321$ & & & $156-412$ & & \\
\hline TG & & & & & & & \\
\hline Mean \pm SD & $228 \pm 67.9$ & $212 \pm 65.9$ & 2.01 & $<0.05$ & $154.8 \pm 43.09$ & 18.8 & $<0.001$ \\
\hline Range & $180-342$ & $165-398$ & & & $97-213$ & & \\
\hline
\end{tabular}

Table-4: Comparison of fasting blood sugar (mg/dl), postprandial blood sugar, and glycated hemoglobin (mg/dl) between the studied groups

\begin{tabular}{|l|l|l|l|l|l|l|l|}
\hline & Group 1 & Group 2 & $\boldsymbol{t}$-Test & $\boldsymbol{p}$ & Group 3 & F (ANOVA) & $\boldsymbol{p}$ \\
\hline FBS & & & & & & & \\
\hline Mean \pm SD & $168 \pm 6.5$ & $127 \pm 4.9$ & 8.6 & $<0.001$ & $90.5 \pm 1.92$ & 88.8 & $<0.001$ \\
\hline Range & $120-220$ & $900-1115$ & & & $70-110$ & & \\
\hline PPBS & & & & & & & \\
\hline Mean \pm SD & $318 \pm 65.8$ & $212 \pm 65.9$ & 8.6 & $<0.001$ & $110.9 \pm 21.8$ & 117.9 & $<0.001$ \\
\hline Range & & & & & & & \\
\hline HbA1c & & & & & & & \\
\hline Mean \pm SD & $11 \pm 1.09$ & $7.87 \pm 1.55$ & & $<0.001$ & $4.9 \pm 0.45$ & 145.9 & $<0.001$ \\
\hline Range & $8-11.5$ & $6.7-11.9$ & & & $4.1-5.9$ & & \\
\hline
\end{tabular}

\section{DISCUSSION}

The results of various studies are positively correlated to circulating leptin concentrations and obesity, despite the antiobesity actions of leptin [6, 7]. Serum leptin levels were observed to be higher in the obese group and positively correlated with body fatness and obesity [8]. This is also in agreement with Adil Omar who found that leptin concentrations were high in both the obese group and the diabetic obese group and showed a direct positive relationship with BMI and waist circumference [9]. Zuo et al. [2] in their population-based study in China reported that leptin was independently associated with all measures of adiposity; however, there was a sex difference in the association of various levels of adiposity measures with leptin, where in men BMI had the strongest association with leptin, whereas in women the strongest association was with triceps skinfold. Martins Mdo et al. [2] concluded that elevated serum leptin, particularly in obese individuals, should be taken as a warning sign of energy imbalance, poor diet, hyperinsulinemia, insulin resistance, or changes in other metabolic risk factors that are strongly associated with CVD and T2DM.Moreover, our study showed that serum leptin levels were statistically significantly raised in patients with metabolic syndrome who were obese (group I), were also high in nonobese T2DM patients (group II), but normal or low in healthy controls. Our study is supported by the findings of a previous study, which observed that elevated leptin levels could confound an association with diabetes. The study concluded that leptin may play a role in the pathophysiology of diabetes, possibly by suppressing insulin secretion [2]. Goya et al. [3] conducted a prospective study in 2007. The study found that increased levels of serum leptin and low adiponectin were associated with increased risk of type 2 diabetes [2, 3]. This was also similar to the study by Anil and colleagues in India who showed an association between increased serum leptin levels and T2DM patients. Leptin levels were significantly high in diabetic patients when compared with age-, sex-, and BMI-matched controls. In the literature there are contradictory data dealing with serum leptin concentrations in type 2 diabetic patients. Our findings are different from those studies reporting lower serum leptin in diabetes. However, our results are comparable with those reporting higher serum leptin in diabetes. It is hard to resolve these differences, but they may be merely related to selection of patient groups, different analytical techniques and type I or II statistical errors or there may be a true hyperleptinemia in type 2 diabetes. It is still unclear how changes in plasma glucose concentrations may affect the release or the metabolism of leptin at a physiological level. Nevertheless, the hyperleptinemia in type 2 diabetes can be explained on the basis of insulin resistance, chronically elevated plasma insulin levels, and diabetic nephropathy. Type 2 diabetes is characterized by insulin resistance, which is positively associated with hyperleptinemia. Insulin induces leptin synthesis in a dose-dependent fashion. Hence, in the presence of insulin resistance and chronic hyperinsulinemia, type 2 diabetics are expected to exhibit hyperleptinemia. Third possibility of increased serum leptin levels could be diabetic renal damage. Type 2 diabetic patients with impaired renal functions had higher serum leptin concentrations than control subjects matched for age, sex, and body fat. It remains to be determined whether the increased serum leptin concentrations in patients with diabetic nephropathy is due to increased production, reduced clearance, or increased leptin resistance. We also found a strong positive correlation between leptin and FBS like the study done by Liu et al. However; it was contradictory to the previous finding of the lack of a positive correlation between plasma glucose and leptin concentrations. 
Table-5: Correlation between serum leptin and other parameters

\begin{tabular}{|c|c|c|c|}
\hline & $\mathbf{r}$ & $\mathbf{p}$ & Significance \\
\hline Age & 0.22 & $>0.05$ & NS \\
\hline BMI & 0.54 & $<0.001$ & HS \\
\hline FBS & 0.67 & $<0.001$ & HS \\
\hline PPBS & 0.40 & $<0.001$ & HS \\
\hline HbA1c & 0.65 & $<0.001$ & HS \\
\hline TC & 0.41 & $<0.001$ & HS \\
\hline TG & 0.45 & $<0.001$ & HS \\
\hline Insulin & 0.54 & $<0.001$ & HS \\
\hline
\end{tabular}

\section{RESULTS}

The study was carried out in a single urban university hospital, so any generalizations are left to the discretion of the reader. We did not exclude the diabetic patients on treatment from study. Antidiabetic treatment may also influence leptin levels, for example, glibenclamide sulfonylurea or insulin therapy, all increase serum leptin concentrations [3, 9]. There are well-known gender differences in leptin levels; we have included both male and female diabetic patients. This might have affected the results. FBS levels were found to be $168.7 \pm 3$ and $126.6 \pm 1 \mathrm{mg} / \mathrm{dl}$ in groups I and II, respectively, as compared with $90.6 \pm 1 \mathrm{mg} / \mathrm{dl}$ in normal, healthy controls. PPBS levels were $318 \pm 6$ in group I, $212.9 \pm 1$ in group II, and $110.9 \pm 8$ in the control group. The mean serum levels of these parameters were significantly higher in group I when compared with group II and with group III $(P<0.001)$ The levels of HbA1c were also observed to be statistically highly significant $(P<0.001)$ in patients with metabolic syndrome $(10 \pm 1 \%)$ when compared with patients without metabolic syndrome $(7.7 \pm 1 \%)$ and with healthy controls (4.9\%). In addition, serum cholesterol and TG levels were observed to be significantly high in patients with metabolic syndrome when compared with patients without metabolic syndrome and with healthy controls $(P<0.001)$. In addition, there was a statistically significant difference between these parameters when group I was compared with group II $(P<0.05)$. Moreover, the mean serum levels of leptin and insulin were significantly high $(P<$ $0.001)$ in group I $(9.4 \pm 2$ for leptin and $116 \pm 9$ for insulin) versus group II (7.7 \pm 1 for leptin and $13.2 \pm 4$ for insulin) and healthy controls $(5.2 \pm 2$ for leptin and $7.2 \pm 1$ for insulin).Correlations between serum leptin and other parameters measured in this study showed no significant differences regarding age of participants, but highly significant differences with regard to BMI, FBS, PPBS, HbA1c, TC, TG, and serum insulin were found $(P<0.001)$.In fact, the most important finding of this study was the positive relationship between serum leptin and serum insulin - that is, serum leptin level increases with increase in serum insulin $(r=0.51, P<$ 0.001). Therefore, in insulin resistance syndrome, where there is a hyperinsulinemic state, we predict that there are high serum leptin levels. In other words, high serum leptin may be a good indicator of the insulin resistance syndrome. In our study, there were significant findings with regard to serum leptin and serum insulin. Serum leptin showed a significant difference between the studied groups $(P<0.001)$. In addition, serum insulin showed the same result $(P<$ 0.001). Serum leptin measured in the studied groups showed a positive relationship with body fat, represented by BMI ( $r=0.52, P<0.001)$, and showed a positive relationship with fasting blood glucose and postprandial blood glucose $(r=0.65, P<0.001$ and $r=$ $0.37, P<0.001$, respectively). Our findings also showed a positive relationship between serum leptin and TC $(r=0.41, P<0.001)$ and TG $(r=0.45, P<$ $0.001)$.The positive relationships between serum leptin and insulin with BMI signify that body fat or obesity plays a role in the development of both hyperleptinemia and hyperinsulinemia presented by insulin resistance syndrome.

\section{CONCLUSION}

In conclusion, in addition to its well-known effects on energy homeostasis, leptin is a hormone that also directly regulates glucose metabolism through its actions via the CNS. Identification of the specific neuronal subsets downstream of leptin action, which link communication between the brain and peripheral tissues to control both hepatic glucose production and glucose uptake, will help facilitate the development of new approaches to diabetes treatment. While there are several hurdles to overcome for targeting the CNS [11] it nonetheless has untapped potential for the treatment of diabetes. Hyperleptinemia could be a new component of insulin resistance syndrome. It can be used as a useful biomarker for diagnosis and early identification of metabolic syndrome. It can be considered as an additional risk factor in patients of T2DM with high BMI (obesity) and dyslipidemia [4].

\section{REFERENCES}

1. Smyth, S., \& Heron, A. (2006). Diabetes and obesity: the twin epidemics. Nature medicine, 12(1), 75-80.

2. Chen, L., Magliano, D. J., \& Zimmet, P. Z. (2012). The worldwide epidemiology of type 2 diabetes mellitus - present and future perspectives. Nature reviews endocrinology, 8(4), 228-236.

3. Economic costs of diabetes in the U.S. in 2007. (2008). Diabetes Care. 31:596-615.

4. Standards of medical care in diabetes2011. (2011). Diabetes Care. 34 (Suppl 1):S11-61 
5. Kahn, S. E., Prigeon, R. L., McCulloch, D. K., Boyko, E. J., Bergman, R. N., Schwartz, M. W., ... \& Palmer, J. P. (1993). Quantification of the relationship between insulin sensitivity and $\beta$-cell function in human subjects: evidence for a hyperbolic function. Diabetes, 42(11), 1663-1672.

6. Devendra, D., Liu, E., \& Eisenbarth, G. S. (2004). Type 1 diabetes: recent developments. Bmj, 328(7442), 750-754.

7. DCCT Research Group. (1988). Weight gain associated with intensive therapy in the diabetes control and complications trial. Diabetes Care, 11(7), 567-573.

8. Diabetes Control and Complications Trial Research Group. (1993). The effect of intensive treatment of diabetes on the development and progression of long-term complications in insulin-dependent diabetes mellitus. New England journal of medicine, 329(14), 977-986.

9. Diabetes Control and Complications Trial Research Group. (1997). Hypoglycemia in the diabetes control and complications trial. Diabetes, 46(2), 271-286.
10. Bluestone, J. A., Herold, K., \& Eisenbarth, G. (2010). Genetics, pathogenesis and clinical interventions in type 1 diabetes. Nature, 464(7293), 1293-1300.

11. Yki-Järvinen, H. (2001). Combination therapies with insulin in type 2 diabetes. Diabetes care, 24(4), 758-767.

12. Riddle, M. C., Rosenstock, J., \& Gerich, J. (2003). The treat-to-target trial: randomized addition of glargine or human NPH insulin to oral therapy of type 2 diabetic patients. Diabetes care, 26(11), 3080-3086.

13. Lebovitz, H. E. (2011). Type 2 diabetes mellituscurrent therapies and the emergence of surgical options. Nature Reviews Endocrinology, 7(7), 408.

14. Schwartz, M. W., \& Porte, D. (2005). Diabetes, obesity, and the brain. Science, 307(5708), 375379.

15. Morton, G. J., \& Schwartz, M. W. (2011). Leptin and the central nervous system control of glucose metabolism. Physiological reviews, 91(2), 389-411. 